\title{
3D Muscle detailed ankle-foot model for finite element analysis
}

\author{
Enrique Morales Orcajo \\ GEMM (Group of Structural Mechanics and Materials Modelling) \\ Instituto de Investigación en Ingeniería de Aragón (I3A). \\ Universidad de Zaragoza, Mariano Esquillor s/n, 50018, Zaragoza, Spain. \\ Tel. +34-976762707, Fax +34-976762043, e-mail: enriquem@unizar.es
}

\begin{abstract}
A three dimensional muscle detailed human anklefoot model was created. Starting from computed tomographies all bones and muscles of the foot were reconstructed. The development process, the model features and the thresholding and smoothing problems are explained. The finite element model developed will be used to study the mechanical performance of each muscle and bone allowing to simulate any pathology, treatment or surgery of the foot.
\end{abstract}

\section{Introduction}

A finite element analysis of biological structures enable to study stresses, strains and displacements inside the organs without surgery, avoiding any risk for the patient and calculating mechanical behaviour of them that would be impossible to test with experimental methods. In order to make the best use of this powerful tool an accurate model of human ankle-foot has been developed. This model can be useful for shoes, insoles and orthotics design. ${ }^{1,2}$ It's also interesting so as to simulate different foot pathologies and study the effect of a specific treatment to alleviate the pain and even simulate possible surgeries to help mainly the surgeon's decision to obtain the best solution. ${ }^{3}$

The most accurate models developed until now identify the bones, cartilage and ligaments in an embedded volume as soft tissue. ${ }^{4}$ Even the models that try to approach some specific muscle only have been simulated with bar elements. ${ }^{5}$ The model proposed identifies the geometry of every foot muscle therefore besides enables to define its behaviour properly. It may also be reported any data about any individual muscle increasing the rate of pathologies can be studied.

The goal of this project is to develop an accurate foot model that enables to predict the biomechanical behaviour of the soft tissue as it has already been occurring during last decade with hard tissue.

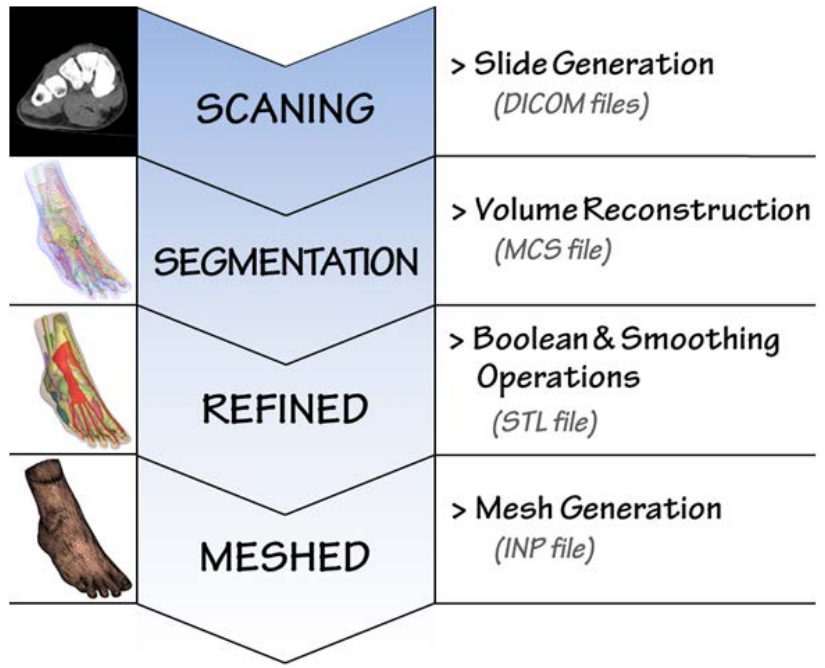

Figure 1 - Modelling methodology diagram

\section{Methodology}

All 3D foot models built up until now follow the same process ${ }^{6}$ and is summarised in Figure 1. Firstly a foot of a 49 year old male with no foot pathology was scanned using a Multi Slice CT: HiSpeed Dual (General Electric). From CT scan were generated 93 cross-sectional cuts with a $3.75 \mathrm{~mm}$ slice distance. The medical images were exported in DICOM format to MIMICS 15.0 for volume reconstruction. This software based on Hounsfield scale was used to distinguish the different material densities obtained from the tomography. Each bone and muscle was segmented individually. After smoothing and Boolean operations, for refining the geometries, all reconstructed volumes were exported in independent STL files. Those files were imported from ABAQUS (ABAQUS 6.11.3, HKS, Pawtucket, RI) where these were meshed. Lastly, the meshes were combined to build the final model. 

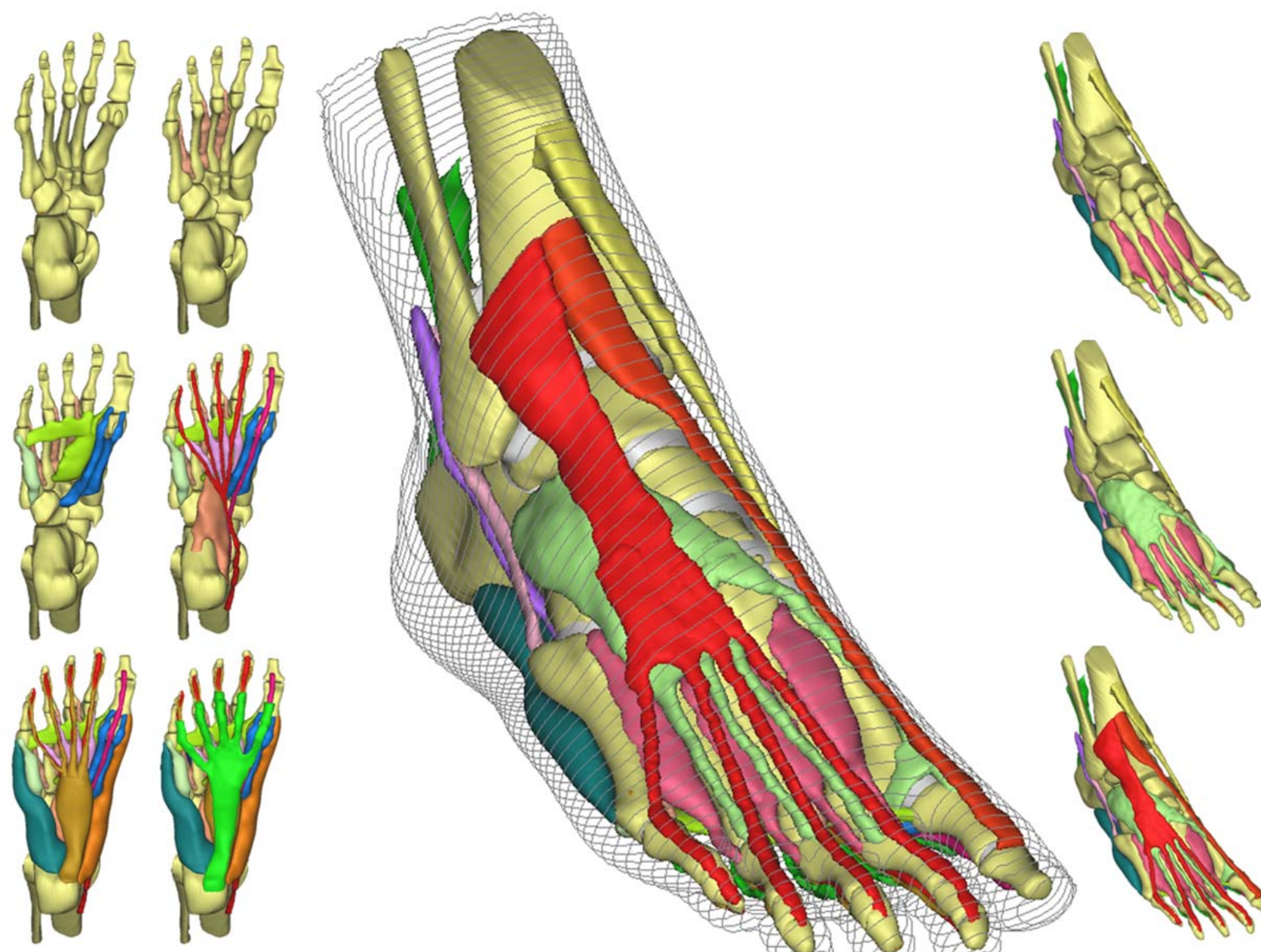

Figure 2 - Volumes generated shown by layers

\section{Model characteristics}

The 3D muscle detailed ankle-foot model gathered 30 bones, 25 muscles, ligaments and cartilages (Figure 2). The bone structure reconstructed was made up of tibia, fibula, talus, calcaneus, cuboid, navicular, 3 cuneiforms, 5 metatarsals, 5 proximal phalanges, 4 middle phalanges, 5 distal phalanges and 2 sesamoids distinguishing between cortical and trabecular bone all of them. Between each bone were created the corresponding cartilage and ligaments. For the muscular system the following were indentified: extensor digitorum longus and brevis, extensor hallucis longus, 4 dorsal and 3 posterior interossei, tibialis anterior and posterior, peroneus longus and brevis, adductor and abductor hallucis, flexor hallucis longus and brevis, flexor digitorum longus and brevis, 4 lumbricals, quadratus plantae, abductor and flexor digiti mini, achilles tendon and plantar fascia. Finally all this geometry was encapsulated in fat tissue. The most interesting of this powerful detailed model was that every single item could be characterized and reported individually, so any disease in feet even complex pathologies involving both soft and hard tissues could be simulated.

\section{Overcome problems}

Thresholding tool in MIMICS was used to define each tissue (cortical bone, trabecular bone, muscle, facia and fat). This method is very useful to distinguish between items with different radiodensities like cortical and trabecular bone or separate bone, muscle-tendon tissue and fat tissue as shown in Figure 3. But defining every muscle was the most difficult challenge of the project. This problem was solved making tight contrasts within the range of muscle Hounsfield units. Flexor and extensor tendons could be defined with this high contrast, others like Achilles tendon was clearly 


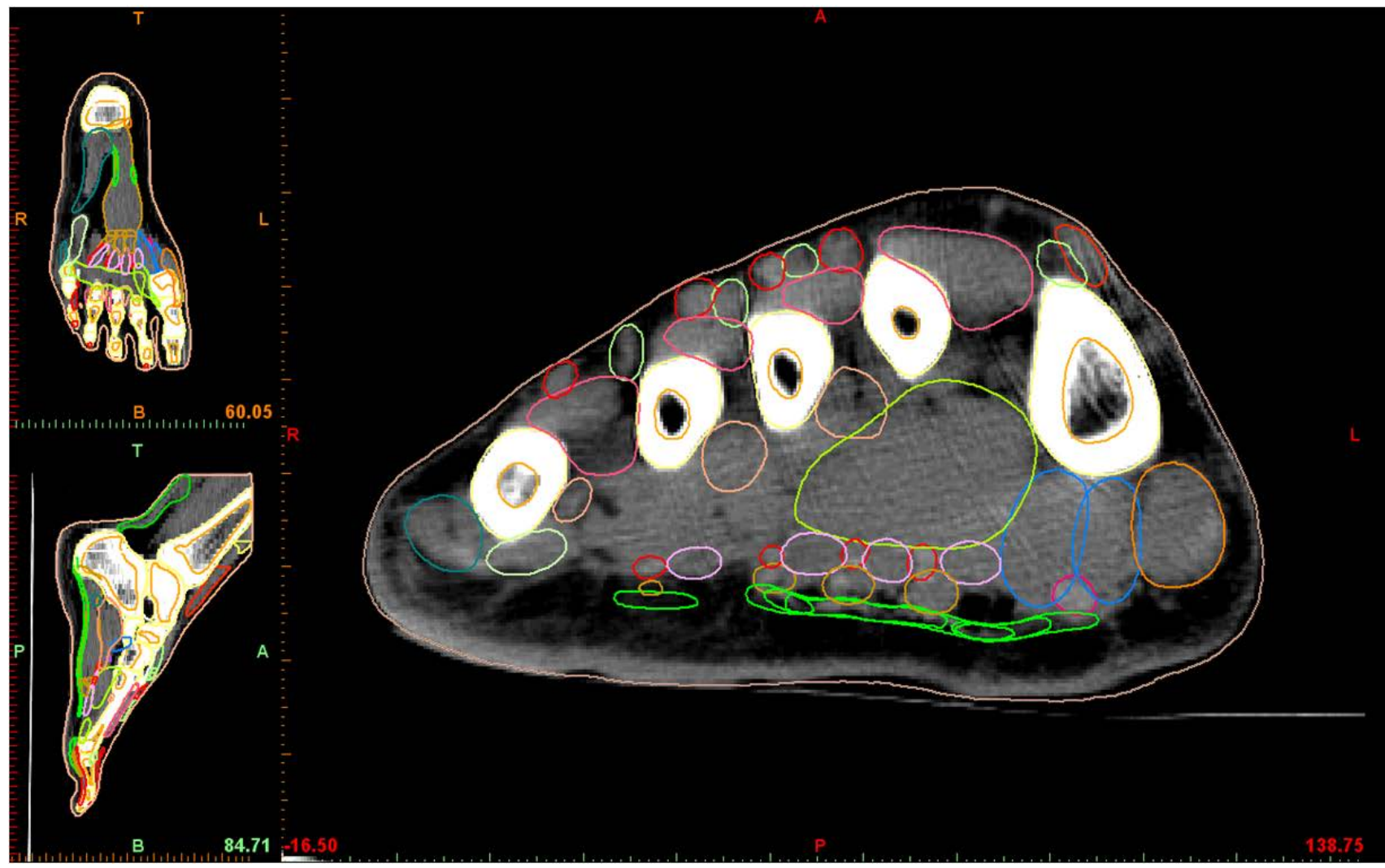

Figure 3 - Items identified in a computed tomography in average contrast. Coronal, sagittal and transverse plane

isolated and with the help of layer distribution of the muscles in midfoot the most difficult segmentation slices could be approached.

Another problem found out during the process was smoothing slim branched tendons like flexors and extensors digitorum tendons, because the smoothing algorithm tried to join the branches from the same muscle. To avoid this disruption it was necessary to segment each branch separately before smoothing, and then join them. 115 volumes have been required to smoothe all geometry correctly.

In order to achieve the most accurate model, all volumes were filled and afterwards all volumes intersected were substracted, thus the trabecular bone was substracted from the cortical bone, and the bone and muscles from the fat tissue, thus avoiding gaps.

\section{REFERENCIAS}

Hsu, Y.-C. et al. Using an optimization approach to design an insole for lowering plantar fascia stress--a finite element study. Annals of biomedical engineering 36, 1345-52 (2008).

Liu, X. \& Zhang, M. Redistribution of knee stress using laterally wedged insole intervention: Finite element analysis of knee-ankle-foot complex. Clinical biomechanics (Bristol, Avon) 28, 61-7 (2013).

Cheung, J. T.-M. \& Nigg, B. M. Clinical Applications of Computational Simulation of Foot and Ankle. SportOrthopädie - Sport-Traumatologie - Sports Orthopaedics and Traumatology 23, 264-271 (2008).

Cheung, J. T.-M., Zhang, M., Leung, A. K.-L. \& Fan, Y.-B. Three-dimensional finite element analysis of the foot during standing - a material sensitivity study. Journal of Biomechanics 38, 1045-1054 (2005).

Chen, W.-M., Park, J., Park, S.-B., Shim, V. P.-W. \& Lee, $\mathrm{T}$. Role of gastrocnemius-soleus muscle in forefoot force transmission at heel rise - A 3D finite element analysis. Journal of biomechanics 45, 1783-9 (2012).

Qiu, T.-X., Teo, E.-C., Yan, Y.-B. \& Lei, W. Finite element modeling of a 3D coupled foot-boot model. Medical engineering \& physics 33, 1228-33 (2011). 